\title{
Aplicação adequada do partograma e o seu impacto na taxa de cesarianas: revisão narrativa
}

\author{
Proper application of the partogram and its impact on the rate of cesarean sections: \\ narrative review \\ Aplicación adecuada del partograma y su impacto en la tasa de cesáreas: revisión \\ narrativa
}

\begin{abstract}
Isabelle Salomão Teixeira Silva1* ${ }^{*}$, Ana Luiza Melo Lima Costa ${ }^{1}$, Julie Kate Ferreira de Oliveira ${ }^{1}$, Nathália Costa de Carvalho², Suzanna Christyna de Souza Lima1 ${ }^{2}$, Marcela Rêda Guimarães ${ }^{3}$, Vanessa Noeme Correa ${ }^{4}$, Verônica Pirâmides Coura Martins de Loyola', Virgínia Pirâmides Coura Martins de Loyola ${ }^{1}$, Adriel Gustavo Lopes ${ }^{1}$.
\end{abstract}

\section{RESUMO}

Objetivo: Analisar se a aplicação adequada do partograma influencia na taxa de cesarianas, bem como, discutir sua importância na escolha da via de parto, incluindo possíveis benefícios da utilização desse instrumento no acompanhamento do trabalho de parto e avaliar a qualidade da assistência ao parto em locais onde o partograma é aplicado regular e corretamente. Revisão Bibliográfica: Trata-se de uma revisão narrativa acerca do uso do partograma e o seu impacto no número de cesarianas. Sabe-se que o partograma tem seu uso recomendado amplamente pela OMS, entretanto seu uso ainda não é amplo em qualquer local. Sendo que, o uso do mesmo mostrou-se de maior impacto em países em desenvolvimento, onde há evidências de que o uso adequado pode diminuir a taxa de cesáreas, fato não observado em regiões de serviços de saúde com maiores recursos econômicos. Considerações finais: Não foi possível estabelecer de forma definitiva uma relação entre a diminuição nas taxas de cesariana e o uso do partograma. O resultado inconclusivo aponta para diferenças relativas ao local de aplicação do partograma, sendo necessário mais dados para confirmar sua relevância como uma ferramenta que contribua para diminuir o número de cesáreas realizadas.

Palavras-chave: Trabalho de parto, Registros médicos, Cesárea.

\section{ABSTRACT}

Objective: To analyze whether the adequate application of the partograph influences the rate of cesarean sections, as well as to discuss its importance in choosing the mode of delivery, including possible benefits of using this instrument in the monitoring of labor and to evaluate the quality of delivery care in places where the partogram is applied regularly and correctly. Bibliographic review: It is a narrative review about the use of the partogram and its impact on the number of cesarean sections. Even though its use is widely recommended by the WHO, its practice has shown to have a greater impact in developing countries, where there is evidence that adequate use of partogram can decrease the rate of cesarean sections, fact not observed in health service regions with greater economic resources. Final considerations: It was not possible to definitively establish a

\footnotetext{
${ }^{1}$ Pontifícia Universidade Católica de Minas Gerais. Betim - Minas Gerais.

*E-mail: isabellesilva554@gmail.com

${ }^{2}$ Universidade Potiguar (UnP). Natal - Rio Grande do Norte.

3 Universidade de Itaúna. Itaúna - Minas Gerais.

${ }^{4}$ Universidade Federal do Piauí (UFPI). Teresina - Piauí.
} 
relationship between the decrease in cesarean section rates and the use of the partogram. The inconclusive results points to differences related to the location of the application of the partogram, requiring more data to confirm its relevance as a tool that contributes to reduce the number of cesarean sections performed.

Key words: Labor obstetric, Medical records, Cesarean section.

\section{RESUMEN}

Objetivo: Analizar si la aplicación adecuada del partograma influye en la tasa de cesáreas, así como analizar su importancia en la elección del modo de parto, incluidos los posibles beneficios de usar este instrumento en el seguimiento del trabajo de parto y evaluar la calidad de la atención del parto. en lugares donde el partograma se aplica de manera regular y correcta. Revisión bibliográfica: Revisión narrativa sobre el uso del partograma. Aunque su uso es ampliamente recomendado por la OMS, esta práctica ha demostrado tener un mayor impacto en los países en desarrollo, donde existe evidencia de que el uso adecuado puede disminuir la tasa de cesáreas, un hecho que no se observa en las regiones de servicios de salud con mayores recursos económicos. Consideraciones finales: No fue posible establecer definitivamente una relación entre la disminución de las tasas de cesárea y el uso del partograma. El resultado no concluyente apunta a diferencias con respecto a la aplicación del partograma, que requiere más datos para confirmar su relevancia como una herramienta que contribuye a reducir el número de cesáreas realizadas.

Palabras clave: Trabajo de parto, Registros médicos, Cesárea.

\section{INTRODUÇÃO}

Emanuel Friedman, em 1951, ao analisar a evolução do trabalho de parto, estabeleceu um padrão de normalidade que permitiu um incremento na qualidade de assistência à parturiente. Dessa forma, foi possível a criação de uma representação gráfica dos eventos esperados em um parto bem sucedido, chamado de partograma. Essa ferramenta é utilizada mundialmente com o intuito de indicar possíveis intercorrências obstétricas e auxiliar no manejo adequado do trabalho de parto (LUCENA TS, et al., 2019). Segundo Montenegro CAB e Rezende Filho J (2017, p. 400), "o partograma é a representação gráfica do trabalho de parto, que torna possível acompanhar a sua evolução, documentar, diagnosticar alterações e indicar a tomada de condutas apropriadas para a correção dos desvios".

O modelo mais conhecido e utilizado compreende duas linhas paralelas com distância indicando um período de $4 \mathrm{~h}$ entre elas, sendo a primeira denominada "linha de alerta" e a segunda, "linha de ação". Preconiza-se que o registro deve ser iniciado na fase ativa do trabalho de parto, ou seja, quando há duas a três contrações generalizadas em 10 minutos e dilatação cervical mínima de $6 \mathrm{~cm}$. A cada exame de toque vaginal, que deve ser realizado com intervalo de $4 \mathrm{~h}$, anota-se a dilatação cervical, a altura da apresentação (documentada em planos de DeLee), a variedade de posição, as condições da bolsa das águas e do líquido amniótico (MONTENEGRO CAB e REZENDE FILHO J, 2017). Recomenda-se também monitorização e registro dos batimentos cardíacos fetais, analgesia, especialmente peridural ou de duplo bloqueio, registro da dose de ocitocina, quando utilizada, procedimentos realizados, a exemplo da amniotomia, e do padrão cardiotocográfico (ZUGAIB M e FRANCISCO RPV, 2016).

Quando a curva de dilatação cervical se processa à esquerda da linha de ação, consideramos a evolução do trabalho sem alterações. Quando essa curva ultrapassa a linha de alerta é necessária maior observação clínica, mas apenas quando cruza a linha de ação pode-se considerar o parto disfuncional, devendo-se realizar alguma intervenção, porém não necessariamente uma conduta cirúrgica. O diagnóstico de falha de progressão é dado se após $4 \mathrm{~h}$ não houver modificação cervical em uma gestante com contrações efetivas e bolsa rota (MONTENEGRO CAB e REZENDE FILHO J, 2017). A Organização Mundial de Saúde (OMS) defende a aplicação do partograma como um mecanismo útil à assistência ao parto de qualidade. No Brasil, a FEBRASGO e o Ministério da saúde reafirmam essa recomendação. Contudo, a análise do estudo Ponce 
JRA, et al. (2011), concluiu que embora o partograma seja de baixo custo e de fácil acesso, observa-se uma baixa taxa de seu registro em prontuário por parte dos profissionais de saúde, podendo reduzir a qualidade do cuidado intraparto.

Além disso, o estudo realizado por Lucena TS, et al. (2019), evidenciou que apenas 40,6\% dos prontuários tinham registro de abertura do partograma e destes, 59,2\% tiveram apenas um único registro, demonstrando falha no processo de monitoramento (LUCENA TS, et al., 2019). Ademais, constatou-se que, no Brasil, a utilização do partograma foi inferior a $45 \%$ dos partos realizados (LEAL MC, et al., 2014).

Conforme uma revisão sistemática realizada pela OMS em 1985, uma taxa média de cesarianas em torno de apenas $10-15 \%$ dos partos é a indicada, uma vez que esse número se associa a uma menor mortalidade materna e neonatal. Contudo, no Brasil, em 2006, 44\% dos partos foram cirúrgicos. No sistema de saúde suplementar esse número foi de $81 \%$ e em 2008 chegou a 84,5\% (LEÃO MRC, et al., 2013). Observa-se, que, por diversos motivos, a ocorrência de cesáreas vem se tornando cada vez mais frequente, tanto nos países desenvolvidos como naqueles em desenvolvimento (OMS, 2015; LAVENDER T, 2012). No ano de 2008, 6.2 milhões de cesáreas desnecessárias foram realizadas no mundo. Dessas, China e Brasil representaram quase $50 \%$ do total de cesarianas sem indicação médica (MASCARELLO KC, et al., 2017).

Diante disso, este artigo tem como objetivo analisar por meio de uma revisão narrativa sobre a aplicação adequada do partograma influência na taxa de cesarianas, bem como, discutir sua importância na escolha da via de parto, incluindo possíveis benefícios da utilização desse instrumento no acompanhamento do trabalho de parto e avaliar a qualidade da assistência ao parto em locais onde o partograma é aplicado regular e corretamente.

\section{REVISÃO BIBLIOGRÁFICA}

\section{Importância do partograma}

O partograma é um gráfico utilizado para monitorar a progressão do trabalho de parto, combinado com a dilatação cervical e a descida da cabeça fetal (VLACHOS G, et al., 2015). Apesar do partograma não ser essencial no manejo intraparto, é importante para prever tendências e alertar situações que exigirão maiores cuidados. Além disso, é um mecanismo que auxilia a troca de turno entre os profissionais, pois fornece informações importantes sobre toda a evolução do trabalho de parto (RANI J, et al., 2014).

É considerado como um artefato que favorece a supervisão da qualidade da assistência e permite uma racionalização no uso de ocitócitos, amniotomia e analgesia, contribuindo assim para a redução da incidência de cesáreas. Além disso, é muito útil no diagnóstico precoce de distocias e para indicar a tomada de condutas apropriadas e evitar intervenções desnecessárias (FEBRASGO, 2010). A ficha completa do acompanhamento do trabalho de parto contém, além dos registros da dilatação cervical e da descida da cabeça fetal, informações como frequência cardíaca fetal, dinâmica uterina, as condições do líquido amniótico, a infusão de líquidos e as especificações de medicamentos usados (BRASIL, 2001).

O papel do partograma determinado por Friedman constitui um método sucinto que permite o registro de informações e a identificação e previsão de anormalidades por comparação com um perfil ideal de progressão (SIZER AR, et al., 2000). Dessa forma, segundo Porto AMF, et al. (2010), o partograma é uma ferramenta que permite um acompanhamento de qualidade do trabalho de parto.

Diversos desenhos introduzem duas linhas ao partograma, chamadas linhas de alerta e ação. Estas linhas auxiliam nas condutas do trabalho de parto normal e também permitem a diferenciação de um parto eutócico do distócico. A linha de alerta é traçada, convencionalmente, no início da fase ativa, pois a abertura do partograma na fase latente pode implicar em intervenções desnecessárias e iatrogênicas.

A linha de ação é traçada após quatro horas, paralelamente à primeira linha. A intervenção médica visando conduzir o parto de uma maneira melhor ou corrigir possíveis anormalidades devem ser realizada quando a curva de dilatação cervical atinge a linha de ação. É importante salientar que a intervenção não é necessariamente cirúrgica (BRASIL, 2001). 
A Organização Mundial de Saúde recomenda a utilização do partograma em todas as gestantes, pois é um mecanismo simples e barato que pode ser aplicado em todo o mundo. A aplicação desse instrumento de registro durante o trabalho de parto é importante para diferenciar o progresso normal do anormal e identificar a necessidade de intervenções (RANI J, et al., 2014).

Nesse contexto, a recomendação do uso do partograma pela Organização Mundial da Saúde (OMS) possui como objetivo melhorar a assistência e reduzir a morbidade e mortalidade materna e fetal, assegurando que as intervenções sejam realizadas apenas quando a evolução for anormal e oferecer riscos à saúde materno-fetal. Por isso seu uso é considerado útil e deve ser estimulado (ROCHA IMS, et al., 2009).

\section{Curvas de Zhang}

O estudo de Zhang J, et al. (2010) propõe um modelo de partograma contemporâneo, após a realização de um estudo observacional retrospectivo multicêntrico nos Estados Unidos, no qual foram selecionadas 62.415 parturientes de 12 centros clínicos. Foram considerados como critérios de inclusão: gravidez a termo com início espontâneo do trabalho de parto, apresentação fetal de vértice, parto vaginal e resultados neonatais normais. Essas gestantes foram divididas conforme o número de paridade $(0,1, \mathrm{e} 2+)$ para a construção de curvas médias de trabalho de parto. Além disso, com o intuito de estimar a duração do trabalho de parto, foi observado a distribuição do tempo necessário para aumentar em $1 \mathrm{~cm}$ a dilatação cervical. Foi calculada a mediana e os percentis 95 . Além desse cálculo, foi analisado o tempo necessário para dilatação alcançar $10 \mathrm{~cm}$ em nulíparas a partir do momento da admissão. Por fim, calculou-se os percentis $95 \mathrm{da}$ duração do trabalho de parto.

Zhang J, et. al. (2010), constatou que conforme aumentava o número de paridade, a idade materna e a massa corporal, maior era a dilatação cervical mediana na admissão. A análise do tempo necessário para progressão da dilatação cervical em $1 \mathrm{~cm}$, indicou que quando há uma dilatação de $4 \mathrm{~cm}$ pode demorar mais de 6 horas e $5 \mathrm{~cm}$ mais de 3 horas. Nas curvas de trabalho de parto para diferentes paridades, constatou-se que em multíparas, após $6 \mathrm{~cm}$ de dilatação cervical, o trabalho de parto é mais acelerado. Além disso, mulheres com mais de 2 gestações entraram na fase ativa mais precocemente. Em concordância, as medianas e os percentis 95 indicaram que até $6 \mathrm{~cm}$ de dilatação o tempo do trabalho de parto é semelhante em todas as gestantes, contudo acima disso as multíparas apresentaram uma evolução superior. Ademais, concluíram que a maioria das gestantes com dilatação cervical maior ou igual à $6 \mathrm{~cm}$ tiveram parto vaginal $\mathrm{e}$ o tempo do primeiro estágio do trabalho de parto foi cerca de 2 horas. Já no segundo estágio do trabalho de parto, os percentis 95 para nulíparas com e sem analgesia peridural foram de 3,6 horas e 2,8 horas, respectivamente.

O partograma sugerido por Zhang J, et. al. (2010) se baseia no partograma de Friedman em relação às curvas de trabalho e valores normais na progressão do trabalho. Contudo, visa reduzir o parto prematuro por cesariana ao concluir que uma grande parte das parturientes podem progredir mais lentamente, não seguindo um padrão consistente na fase ativa. Além disso, sugeriu que o marco de início da fase ativa do trabalho de parto seja por volta de $6 \mathrm{~cm}$ de dilatação cervical. Diante desse estudo, o autor definiu como prisão do parto quando, após $6 \mathrm{~cm}$ de dilatação, o tempo para progressão em $1 \mathrm{~cm}$ ser superior a 4 horas. Por fim, esse partograma não utiliza a linha de alerta e a linha do percentil 95 equivale a linha de ação, contudo é uma linha exponencial.

\section{Partograma na escolha da via de parto}

Uma das vantagens associadas ao partograma é a sua praticidade e facilidade no uso, além de ser uma ferramenta acessível pelo baixo custo. Esses aspectos contribuem para sua aplicação, porém foi observado também que o amplo uso do partograma pode levar a uma restrição na gama de intervenções que o obstetra poderia utilizar caso julgasse necessário, diminuindo sua autonomia e restringindo suas ações ao que é recomendado de forma generalizada no partograma. Portanto, existe um risco do partograma ter um impacto negativo em um tratamento individualizado, o que pode interferir na discussão com a gestante de uma via de parto, causando até impactos psicológicos ao não possibilitar um tratamento personalizado (LAVENDER T, et al., 2013). 
Ainda analisando a possibilidade do partograma engessar as ações no trabalho de parto, para Lavender T, et al. (2013) e Porto AMF, et al. (2010), existe a preocupação de que o partograma possa gerar intervenções desnecessárias. Trata-se de uma ferramenta a qual possui uma aplicação generalizada para todo trabalho de parto, desconsiderando, assim, aspectos individuais como raça, nível socioeconômico e cultural, podendo levar ao uso de artifícios como ocitocina, ruptura de membranas, em circunstâncias que, caso fosse possível flexibilizar o momento da ação, poderiam ser evitadas. Além disso, Rani J, et al. (2014) considera que o partograma ao generalizar a evolução do trabalho de parto pode limitar a prática clínica e reduzir a possibilidade do cuidado individualizado a cada gestante.

Em contrapartida, um estudo realizado por Vlachos G, et al. (2015), apresentou resultados demonstrando que a utilização do partograma é um importante mecanismo de auxílio na escolha da via de parto ao associar a falha na descida da cabeça fetal e a parada da dilatação cervical com um aumento das taxas de cesariana.

\section{Qualidade da assistência ao parto em países que fazem uso do partograma}

Um grande estudo randomizado controlado feito por Lavender T, et al. (1999), foi realizado com 615 primíparas e teve como desfechos primários, taxa de cesárea e satisfação materna. A randomização foi realizada com linhas de ação traçadas em 2, 3 e 4 horas à direita da linha de alerta. Os resultados mostram que houve diferenças nas taxas de intervenção obstétrica e cesariana entre os grupos estudados, porém não obtiveram significância estatística.

Os resultados apontaram que as mulheres alocadas com a linha de ação traçada em 2 horas ficaram consideravelmente mais satisfeitas do que as dos grupos de 3 e 4 horas, apesar de terem maior intervenção obstétrica. Outro resultado imprevisível sugere que apreensão de um trabalho de parto mais demorado sem intervenção pode causar maior insatisfação para a mãe quando se compara à identificação precoce de padrões anormais de parto com intervenções resolutivas (LAVENDER T, et al., 1999).

Rani J, et al. (2014), ao analisar diferentes estudos, constatou que nos locais com baixos recursos financeiros, observa-se que a utilização do partograma permite uma melhor comunicação entre os profissionais da assistência ao parto e melhor distribuição dos recursos disponíveis.

Assim, nos países subdesenvolvidos, o partograma se identificou como uma ferramenta importante no gerenciamento do trabalho de parto, permitindo reduzir a morbimortalidade materna e melhorar os resultados maternos e fetais (RANI J, et al., 2014).

Além disso, estudos conduzidos no México e África observaram redução na realização de cesáreas quando o partograma era utilizado para identificar a necessidade de intervenções precoces durante o trabalho de parto. Esta deteç̧ão precoce relacionava-se aos trabalhos de parto considerados longos, que aumentariam o risco de infecções e hemorragias, o que é mais comum em países com recursos reduzidos nos serviços de saúde (LAVENDER T, et al., 2013).

Segundo Porto AMF, et al. (2010), especificamente em países de baixa renda, foi encontrada a redução da taxa de cesárea e, com isso, destacou a utilidade do partograma em populações pobres com difícil acesso à saúde.

Ademais, outro estudo analítico conduzido por Ponce JRA, et.al. (2011) no Departamento de Ginecologia e Obstetrícia do hospital Dos de Mayo, localizado em Lima, Peru, evidenciou que, em países em desenvolvimento, a utilidade do partograma está associada também ao seu baixo custo e acessibilidade.

\section{O impacto do partograma na taxa de cesáreas}

Uma revisão de literatura realizada por Porto AMF, et al. (2010) analisou as evidências disponíveis sobre a assistência aos primeiros períodos de parto. Dentre as revisões, um estudo multicêntrico patrocinado pela OMS, publicado em 1994, foi encontrado redução da taxa de cesariana relacionada à implementação do partograma.

Além desse artigo, Porto AMF, et al. (2010) também abordaram uma meta-análise na qual foi avaliado o seu uso. Esta demonstrou não haver diferença nas taxas de cesarianas relacionadas ao uso do partograma 
e concluiu ser necessário realização de mais ensaios clínicos para estabelecer a eficácia do uso do partograma no acompanhamento do trabalho de parto.

Na Índia, no Patna Medical College, um estudo prospectivo controlado e randomizado com 400 primigestas de alto risco, na fase ativa do parto, durante o período de outubro de 2006 a agosto de 2008, teve o intuito de analisar o papel do partograma nas gestações de alto risco em relação ao modo de parto e ao momento de intervenção ativa. Os casos foram estratificados em parto induzido e em início do trabalho de parto espontâneo. Os resultados não demonstraram diferença estatisticamente significativa entre os dois grupos estudados (RANI J, et al., 2014).

Dessa forma, Rani J, et al. (2014) concluíram que o partograma não teve um impacto significativo no manejo de gestações de alto risco, pois a sua aplicação não alterou a duração do trabalho de parto, a taxa de cesarianas, a taxa de partos vaginais operatórios e o resultado perinatal.

Em contrapartida, um estudo retrospectivo com 478 gestantes no hospital universitário de Alexandrópolis, na Grécia avalia a eficácia do partograma clássico da OMS em relação a um novo modelo de partograma como potencial redutor nas taxas de cesarianas. A população do estudo foi dividida em 2 grupos, conforme 0 tipo de partograma utilizado para monitoramento do trabalho de parto. Assim, 181 partos foram monitorados com o partograma clássico, que possui 2 linhas, com a avaliação da dilatação cervical e da linha de ação a cada uma hora. Ademais, 296 partos foram submetidos ao novo modelo de partograma que possui uma linha que depende de três parâmetros: dilatação cervical, linha da descida da cabeça fetal e ações. Os parâmetros registrados nos 2 grupos foram: dilatação cervical, frequência cardíaca fetal, pressão arterial, temperatura materna, resultado fetal pós natal, características das gestantes e o tempo em minutos desde o momento da decisão até o momento da intervenção (VLACHOS G, et al., 2015).

No partograma clássico, observou-se uma mediana: 318,4 $\pm 10,4$ min na duração do trabalho de parto e 89 partos cesariana. No novo partograma, a mediana da duração do trabalho de parto foi de $246,56 \pm 8,28$ min e 49 partos cesariana. Contudo, ao analisar o tempo de progresso do trabalho de parto nas mulheres que foram submetidas à cesariana, a aplicação do novo partograma teve uma mediana superior $(512,00 \pm 16,61$ min) do que o partograma clássico da OMS (429,17 $\pm 15,34 \mathrm{~min})$, ou seja, $p=000,1$; intervalo de confiança de $95 \%$ da diferença (33,97-131,69 min). Desta maneira, o estudo evidenciou haver benefícios potenciais na redução da incidência do trabalho de parto prolongado e das taxas de cesarianas com a aplicação do novo partograma, quando comparado com o partograma clássico da OMS ( $<<0,001)$ (VLACHOS G, et al., 2015).

Estudo realizado pelo Departamento de Ginecologia e Obstetrícia da Universidade de Toronto, no Canadá de julho de 1997 a dezembro de 1999 e publicado em 2007 realizou uma análise prospectiva do uso do partograma como ferramenta de cabeceira no gerenciamento do trabalho de parto e redução das taxas de cesáreas e intervenções obstétricas. Participaram deste estudo mulheres nulíparas, com 36 a 42 semanas de gestação e com uma gravidez única com apresentação cefálica que foram abordadas pela equipe de pesquisa no início do trabalho de parto em relação à inscrição no estudo. As mulheres que optaram por participar do estudo foram divididas aleatoriamente em um dos dois grupos: o "grupo padrão", cujo progresso no trabalho de parto foi registrado em anotações escritas, ou o "grupo partograma", cujo progresso no trabalho de parto foi registrado em um partograma, bem como nas notas padrões (WINDRIM R, et al., 2007).

Segundo Windrim R, et al. (2007) não houve diferença significativa na taxa de realização de cesáreas entre os dois grupos no resultado primário com $25 \%$ das mulheres no grupo padrão e $24 \%$ no grupo partograma. Também não havia diferenças significativas no parto vaginal espontâneo ou operatório entre os dois grupos. No grupo partograma, a taxa de conclusão do partograma foi de $81 \%$. Os outros $19 \%$ dos partogramas apresentaram um número variável de omissões.

As taxas de intervenções intraparto também foram as mesmas em cada grupo. Sendo assim, esse estudo demonstrou que a adição de um partograma intraparto não resultou em nenhuma diferença mensurável na incidência de intervenções intraparto ou na duração do trabalho de parto.

Um estudo bibliográfico realizado na base de dados do Cochrane Library analisou seis estudos clínicos envolvendo 7706 mulheres, dois estudos que comparavam uso de partograma e não uso de partograma, além 
de estudos que avaliaram estilos diferentes de partograma. Similarmente, o resultado obtido revelou que não houve evidência de nenhuma diferença entre uso e não uso de partograma na taxa de cesárea ou de parto vaginal (LAVENDER T, et al., 2013).

\section{CONSIDERAÇÕES FINAIS}

A eficácia da aplicação do partograma é controversa, não sendo possível concluir o real impacto na rotina clínica. A análise do estudo permite concluir que os benefícios desse instrumento variam conforme o local, sendo observado um elevado potencial em melhorar a qualidade do trabalho de parto em regiões com baixos recursos financeiros. Além disso, a utilização desse instrumento como um fator de restrição à autonomia médica em tomar decisões baseadas na experiência clínica e educacional, ao generalizar a evolução do trabalho de parto, deve ser melhor avaliada. Diante dos resultados inconclusivos em relação ao impacto nas taxas de cesariana e aos reais benefícios do partograma, constata-se a necessidade de mais estudos nessa temática, incluindo testes de eficácia de novos modelos de partograma que têm se mostrado benéficos, inclusive na redução das taxas de cesarianas, para auxiliar os profissionais no manejo adequado intraparto.

\section{REFERÊNCIAS}

1. BRASIL. Ministério da Saúde. Secretaria de Políticas da Saúde. Parto, aborto e puerpério: assistência humanizada à mulher. Brasília, 2001.

2. FEBRASGO. Manual de Orientação Assistência ao Abortamento, Parto e Puerpério. Federação Brasileira das Associações de Ginecologia e Obstetrícia (FEBRASGO), 2010.

3. LAVENDER T, et al. Managing labor using partograms with different action lines: a prospective study of women's views. Birth, 1999; 26(2):89-96.

4. LAVENDER T, et al. Caesarean Section for Non-Medical Reasons at Term. Cochrane Database of Systematic Reviews, 2012; 3.

5. LAVENDER T, et al. Effect of partogram use on outcomes for women in spontaneous labour at term. Cochrane Database of Systematic Reviews, 2013; 7(4)1-79.

6. LEAL MC, et al. Intervenções obstétricas durante o trabalho de parto e parto em mulheres brasileiras de risco habitual. Cad. Saúde Pública, 2014; 30(1):17-32.

7. LEÃO MRC, et al. Reflexões sobre o excesso de cesarianas no Brasil e a autonomia das mulheres. Ciência \& Saúde Coletiva, 2013; 18(8):2395-2400.

8. LUCENA TS, et al. Análise do preenchimento do partograma como boa prática obstétrica na monitorização do trabalho de parto. Rev Fun Care Online, 2019; 11(1):222-227.

9. MASCARELLO KC, et al. Complicações maternas e cesárea sem indicação: revisão sistemática e meta-análise. Revista de Saúde Pública, 2017; 51-105.

10. MONTENEGRO CAB, REZENDE FILHO J. Rezende obstetrícia. 13. ed. Rio de Janeiro: Guanabara Koogan, 2017; $1104 \mathrm{p}$.

11. ORGANIZAÇÃO MUNDIAL DA SAÚDE (OMS). Declaração da OMS sobre taxas de cesáreas. Geneva, 2015.

12. PONCE JRA, et al. Un programa de capacitación previo al inicio de la práctica profesional de médicos peruanos mejora sus conocimientos sobre atención de parto y partograma. Rev. bras. educ. med., 2011; 35(2):186-192.

13. PORTO AMF, et al. Assistência ao primeiro período do trabalho de parto baseada em evidências. Femina, 2010; 38(10):528-537.

14. RANI J, et al. Role of partogram in high risk pregnancies: an experience at a tertiary centre.: an experience at a tertiary centre. Archives Of Gynecology And Obstetrics, 2014; 291(1):73-78.

15. ROCHA IMS, et al. O partograma como instrumento de análise da assistência ao parto. Revista da Escola de Enfermagem da USP, 2009; 43(4):880-888.

16. SIZER AR, et al. A second-stage partogram. Obstetrics \& Gynecology, 2000; 96(5):678-683.

17. VLACHOS G, et al. The effect of the use of a new type of partogram on the cesarean section rates. Journal Of The Turkish German Gynecological Association, 2015; 16(3):145-148.

18. WINDRIM R, et al. A Randomized Controlled Trial of a Bedside Partogram in the Active Management of Primiparous Labour. Journal Of Obstetrics And Gynaecology, 2007; 29(1):27-34.

19. ZHANG J, et al. Contemporary Patterns of Spontaneous Labor With Normal Neonatal Outcomes. Obstetrics \& Gynecology, 2010; 116(6):1281-1287.

20. ZUGAIB M, FRANCISCO RPV. Zugaib obstetrícia. 3. ed. Barueri: Manole, 2016; 1424 p. 